\title{
Bilateral Distal Clavicle Nonunion: A Case Report
}

\author{
Mouad Guenbdar Mourad Bennani Taoufik Cherrad Hassan Zejjari \\ Jamal Louaste Larbi Amhajji \\ Department of Orthopaedics \& Traumatology, Military Hospital Moulay Ismail, Meknes, \\ Morocco
}

Keywords

Distal clavicle $\cdot$ Bilateral $\cdot$ Nonunion

\begin{abstract}
Bilateral clavicle nonunion is extremely rare (4 published cases), and no case of bilateral distal clavicle nonunion has been reported in the literature. We present the case of a 75 -year-old patient followed up for chronic obstructive pulmonary disease, presenting a bilateral fracture of the distal clavicle type "Neer 1" following a road traffic accident and orthopedically treated. After 1 year, the patient complained of mild pain at both shoulders occasionally in cold weather and during an unusual effort without neurovascular symptom associated. The shoulder radiographs showed bilateral distal clavicle nonunion. We chose a conservative treatment because of the mild symptomatology, advanced age, and limited functional demands. The functional outcome was satisfactory. The therapeutic decision in the treatment of the bilateral distal clavicle nonunion is difficult, whereas the therapeutic indications are based on symptomatology, functional impairment, age, comorbidities, and functional demands.
\end{abstract}

\section{Introduction}

Bilateral clavicle fractures are extremely uncommon, the incidence being $<0.5 \%$ of all the clavicle fractures [1]. Distal clavicle fractures without instability consolidate often after conservative treatment, with satisfactory functional outcomes [2].

Four cases of bilateral clavicle nonunion reported in the literature were located in the mid-third of the clavicle [3-6]. Bilateral clavicle nonunion can be treated operatively or conservatively. We report the first case of bilateral distal clavicle nonunion treated conservatively in an adult.

\section{Karger!}




\section{Case Reports in Orthopedic Research}

\section{Case Report}

A 75-year-old man, right-handed, followed up for a chronic obstructive pulmonary disease, was a victim of a road traffic accident that resulted in direct trauma in both shoulders. He has no occupation. He suffered from pain and functional impotence of both shoulders. The physical examination found pain in the palpation of both clavicles, and the piano key sign was absent. The Neurovascular examination of both upper limbs was normal. His chest and shoulder radiographs revealed bilateral distal clavicle fractures "type Neer 1" (Fig. 1). These were treated with figure-of-eight bandage for 6 weeks. He missed the follow-up consultation planned after 6 weeks of immobilization.

One year later, the patient complained of mild pain at both shoulders occasionally in cold weather and during an unaccustomed effort without associated neurovascular symptoms. He reported that the bandage has been applied for 6 weeks. His physical examination stated that there was no obvious deformity of the both shoulders, but the patient complained of a pain on palpation of both distal clavicles. It was also found that the range of motion was limited (right shoulder: flexion $100^{\circ}$, extension $40^{\circ}$, external rotation $50^{\circ}$, and internal rotation $90^{\circ} /$ left shoulder: flexion $110^{\circ}$, extension $40^{\circ}$, external rotation $60^{\circ}$, and internal rotation 100 ). The constant shoulder score was 60 for the right side and 65 for the left one. Furthermore, radiographs of both shoulders showed bilateral distal clavicle nonunion and the wide left coracoclavicular interval (Fig. 2). Nonoperative treatment was prescribed: nonsteroid antiinflammatory for local application and oral paracetamol to the onset of pain, and we recommended him to avoid excessive and unusual stress on the shoulder girdle.

A review after 2 years following his initial injury, we noted a satisfaction of the patient and a full range of motion without neurovascular symptom associated. On the same examination, the Constant shoulder score was 83 for the right shoulder and 85 for the left shoulder.

\section{Discussion}

Bilateral fractures of the clavicle were very uncommonly reported in the literature. The mechanism of sustaining bilateral clavicle fractures were direct blows to both shoulder girdles, compressive force across both shoulder girdles, direct trauma on 1 side, and indirect violence in a subsequent fall on the other or 2 sequential episodes of direct trauma to the shoulders [1].

Fig. 1. Radiographs showing bilateral distal clavicle fractures "type Neer 1 ".

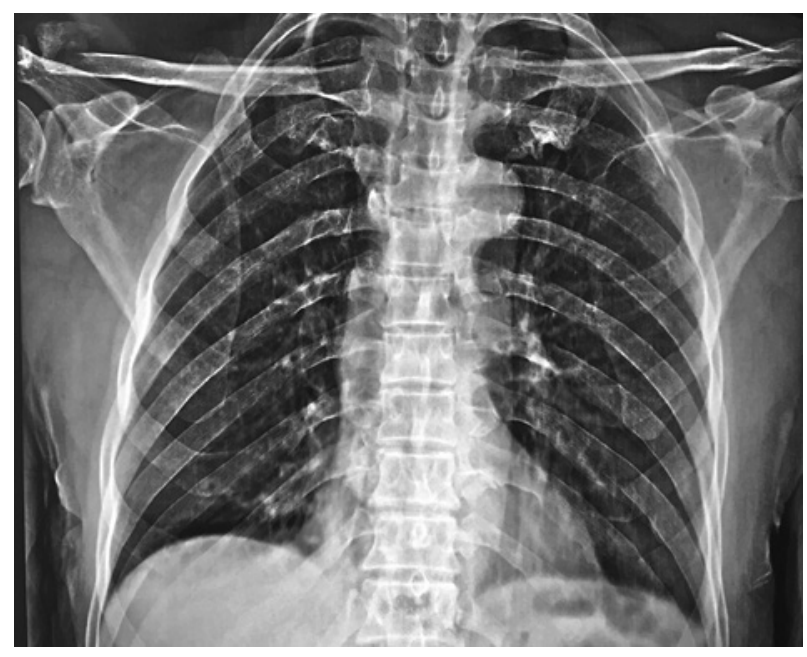




\section{Case Reports in Orthopedic Research}

\begin{tabular}{l|l}
\hline Case Rep Orthop Res 2021;4:152-156 \\
\hline DOI: 10.1159/000515564 & $\begin{array}{l}\text { (c) 2021 The Author(s). Published by S. Karger AG, Basel } \\
\text { www.karger.com/cio }\end{array}$ \\
\hline
\end{tabular}

Guenbdar et al.: Bilateral Distal Clavicle Nonunion

Fig. 2. Radiographs after 1 year showing bilateral distal clavicle nonunion.

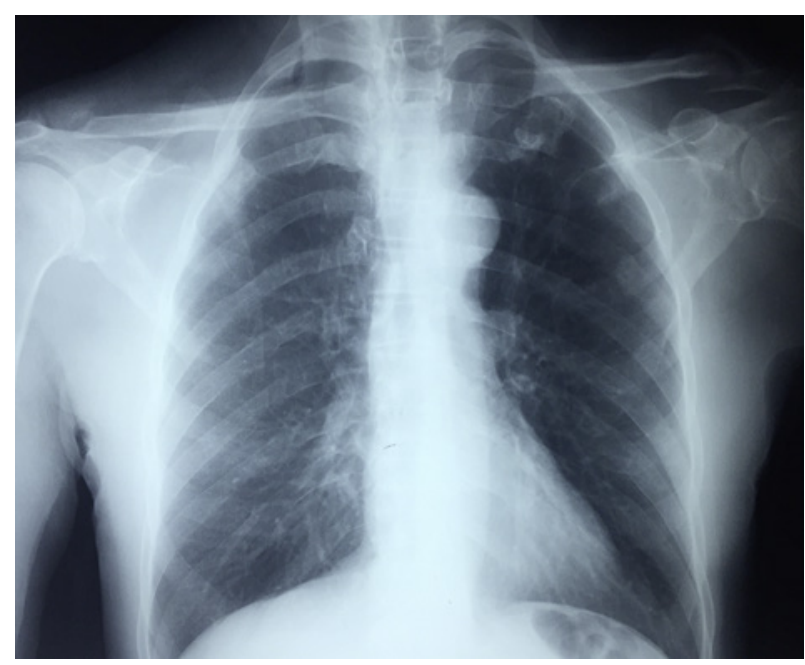

Displaced fractures of the lateral end of the clavicle have a high rate of union, and the functional outcomes are good after nonoperative treatment [7]. But therapeutic indications of the bilateral fractures were not clear and remain a debatable issue [1].

Marya et al. [8] reported 5 cases of bilateral fractures of the clavicle healed after nonoperative treatment. van den Bout et al. [1] suggest an open reduction internal fixation by locking plates to limit the duration of functional disability and to improve the ventilator function of severe associated chest injuries. Several risk factors of nonunion have been reported such as [9-11] age, gender, comminution, shortening $>15 \mathrm{~mm}$, and unstable distal fracture. Although the patient had risk factors of nonunion such as age, chronic smoking, and comminution, we chose a nonoperative method to treat the fractures because of the antecedents of the patient, high anaesthetic risk, and absence of the displacement (type Neer 1).

Villa et al. [12] suggest that an asymptomatic distal clavicle nonunion should not require operative treatment; however, if it is symptomatic, superior or double-plate fixation and bone grafting are indicated. Santolini et al. [13] treated a symptomatic distal clavicle nonunion with a hook plate without bone grafting.

No case of bilateral distal clavicle nonunion has been reported. Bilateral clavicle nonunion is extremely uncommon; only 4 cases have been published in the literature: the age of these cases was inferior to 75 years and clavicular nonunion was localized in mid-third [3-6]. One case among them has been treated conservatively with good functional outcomes [3]. In the 3 other cases reported, nonunion has been very symptomatic and pain was permanent with functional impotence.

These cases had been treated operatively by open reduction internal fixation with bone grafting. Different types of plates were used for fixation: anteroinferior locking compression plating [5] and dynamic compression plate [4]. All cases of bilateral clavicle nonunion treated operatively were healed with good functional outcomes.

In our case, pain was mild and occasional and physical examination was normal (full range of motion and absence of neurovascular symptoms). We treated our patient conservatively because the nonunion did not cause functional impairment and his functional demands were limited, with high anaesthetic risks and advanced age.

In conclusion, bilateral distal clavicle nonunion in adults is extremely uncommon. The choice between conservatively treatment and surgery was difficult because no clear consensus has been established on the management of the distal or bilateral clavicle nonunion, and no 


\section{Case Reports in Orthopedic Research}

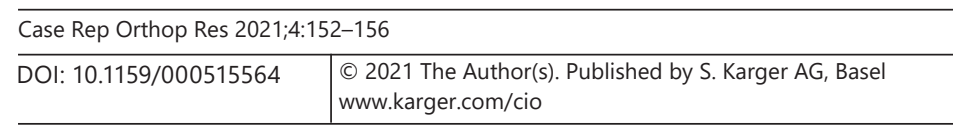

Guenbdar et al.: Bilateral Distal Clavicle Nonunion

similar case has been previously published. Symptomatology, functional impairment, age, comorbidities, and functional demands determine the treatment strategy.

We suggest treating operatively the symptomatic bilateral distal clavicle nonunion. On the other hand, the conservative treatment was sufficient for asymptomatic nonunion without functional impairment, and especially in older patients oligosymptomatic with comorbidities and limited functional demands.

\section{Statement of Ethics}

Written informed consent was obtained from the patient for publication of this case report and for any accompanying images. No identifiers are included in this article pertaining to the patient's identity.

\section{Conflict of Interest Statement}

The authors have no conflicts of interest to disclose.

\section{Funding Sources}

The authors declare that there was no financial support or sponsorship for this study.

\section{Author Contributions}

Mouad Guenbdar and Mourad Bennani contributed to the writing and editing of this article. Mouad Guenbdar and Taoufik Cherrad contributed to the submission process. Hassan zejjari and Jamal Louaste contributed to referencing of this article. Larbi Amhajji contributed to critical revision of the manuscript.

\section{References}

1 van den Bout HET, Snyckers CH. Bilateral clavicle fractures: a case report and review of the literature. SA Orthop J. 2011 Jan;10(2):56-66.

2 Ockert B, Wiedemann E, Haasters F. [Distal clavicle fractures. Classifications and management]. Unfallchirurg. 2015 May;118(5):397-406.

3 Hargan B, Macafee AL. Bilateral pseudarthrosis of the clavicles. Injury. 1981 Jan;12(4):316-8.

4 Kloen P. Bilateral clavicle non-unions treated with anteroinferior locking compression plating (LCP): a case report. Acta Orthop Belg. 2004 Dec;70(6):609-11.

5 Mullett H, Laing A, Curtin W. Successful operative treatment of bilateral clavicle non-union. Injury. 2001 Jan; 32(1):69-70.

6 O'Connor D, Kutty S, McCabe JP. Long-term functional outcome assessment of plate fixation and autogenous bone grafting for clavicular non-union. Injury. 2004 Jun;35(6):575-9.

7 Khan LA, Bradnock TJ, Scott C, Robinson CM. Fractures of the clavicle. J Bone Joint Surg Am. 2009 Feb;91(2): 447-60.

8 Marya KM, Yadav V, Kochar H. Bilateral clavicle fractures. Hong Kong J Orthop Surg. 2002;6(1):39-41.

9 Martetschläger F, Gaskill TR, Millett PJ. Management of clavicle nonunion and malunion. J Shoulder Elbow Surg. 2013 Jun;22(6):862-8.

10 Zlowodzki M, Zelle BA, Cole PA, Jeray K, McKee MD, Evidence-Based Orthopaedic Trauma Working Group. Treatment of acute midshaft clavicle fractures: systematic review of 2144 fractures: on behalf of the evidencebased Orthopaedic Trauma Working Group. J Orthop Trauma. 2005 Aug;19(7):504-7. 
11 Robinson CM, Court-Brown CM, McQueen MM, Wakefield AE. Estimating the risk of nonunion following nonoperative treatment of a clavicular fracture. J Bone Joint Surg Am. 2004 Jul;86(7):1359-65.

12 Villa JC, van der List JP, Gausden EB, Lorich DG, Helfet DL, Kloen P, et al. Plate fixation and bone grafting of distal clavicle nonunions: radiologic and functional outcomes. Arch Orthop Trauma Surg. 2016 Nov;136(11): 1521-9.

13 Santolini E, Stella M, Sanguineti F, Felli L, Santolini F. Treatment of distal clavicle nonunion with and without bone grafting. Injury. 2018 Dec;49 Suppl 4:S34-8. 